\title{
TOPOLOGIES ON THE GROUP OF INVERTIBLE TRANSFORMATIONS
}

\author{
MACIEJ BURNECKI and ROBERT RAŁOWSKI \\ Institute of Mathematics and Computer Science, Wrockaw University of Technology \\ Wybrzeże Wyspiańskiego 27, 50-370 Wrocław, Poland \\ E-mail:Maciej.Burnecki@pwr.wroc.pl,Robert.Ralowski@pwr.wroc.pl
}

\begin{abstract}
We enlarge the amount of embeddings of the group $G$ of invertible transformations of $[0,1]$ into spaces of bounded linear operators on Orlicz spaces. We show the equality of the inherited coarse topologies.
\end{abstract}

1. Introduction. This paper is another step in discussing definitions of coarse topologies on the group of invertible transformations. The consideration was started in [CK] and then continued in [B1], [B2].

Let $m$ denote the Lebesgue measure on $[0,1]$. The group $G$ of all invertible transformations of $[0,1]$ consists of functions $\tau:[0,1] \rightarrow[0,1]$ such that $\tau$ is invertible (injective and surjective) and both $\tau, \tau^{-1}$ are Borel measurable and nonsingular $(m(A)=0$ iff $\left.m\left(\tau^{-1}(A)\right)=0\right)$. Maps equal almost everywhere are identified.

We say that $\phi:[0, \infty) \rightarrow[0, \infty)$ is an Orlicz function if it is convex and $\phi(x)=0$ iff $x=0$. We say that an Orlicz function $\phi$ satisfies the condition $\Delta^{\prime}$ globally if there exists $c>0$ such that $\phi(x y) \leq c \phi(x) \phi(y)$ for $x, y \in[0, \infty)$. In this work Orlicz spaces are equipped with the Luxemburg norm $\|\cdot\|_{\phi}$. The same symbol is used for norms of operators on Orlicz spaces.

If $\phi$ is an Orlicz function which satisfies the condition $\Delta^{\prime}$ globally then the formula

$$
T_{\tau}^{(\phi)}(f)=\left(f \circ \tau^{-1}\right)\left(\phi^{-1} \circ \omega_{\tau}\right),
$$

where $f \in L^{\phi}(m), \tau \in G$ and $\omega_{\tau}=\frac{d\left(m \circ \tau^{-1}\right)}{d m}$ denotes the Radon-Nikodym derivative of the measure $m \circ \tau^{-1}$ with respect to $m$, gives a bounded linear operator on the Orlicz space $L^{\phi}(m)$. The inherited topologies from the strong operator topology on $\mathcal{L}\left(L^{\phi}(m)\right)$ coincide on $G$ - it was proved in [CK] for $L^{p}$-spaces and then generalized in [B1], B2] for Orlicz spaces.

2010 Mathematics Subject Classification: Primary 46E30; Secondary 47B37.

Key words and phrases: invertible transformation, Orlicz space, strong operator topology. The paper is in final form and no version of it will be published elsewhere. 
We generalize formula (1) and obtain more sets of bounded linear operators on $L^{\phi}(m)$. We show equality of the inherited topologies.

Basic information on Orlicz spaces can be found in $\mathrm{KR}$ and $\mathrm{RR}$. An interesting study of generating $G$ in the inherited topology by two transformations is published in $\mathrm{G} 2$ and $\left[\mathrm{P}\right.$. A characterization of $L^{p}$-spaces with a help of operators generated by invertible transformations is given in $\mathrm{B} 3$.

2. The extension. We start with a generalized definition of the embedding.

Definition 2.1. Let $h:[0, \infty) \rightarrow[0, \infty)$ be a Borel measurable function and $\tau \in G$. We denote by $\omega_{\tau}$ the Radon-Nikodym derivative of the measure $m \circ \tau^{-1}$ with respect to $m$. We introduce an operator $T_{\tau}^{(h)}: L^{0}(m) \rightarrow L^{0}(m)$, where $L^{0}(m)$ stands for the set of all real $m$-measurable functions, by the formula

$$
T_{\tau}^{(h)}(f)=\left(f \circ \tau^{-1}\right)\left(h \circ \omega_{\tau}\right) \text { for } f \in L^{0}(m) .
$$

The section of the operator $T_{\tau}^{(h)}$ to an Orlicz space $L^{\phi}(m)$ is denoted by $T_{\tau}^{(\phi, h)}$.

Proposition 2.2. Let $\tau \in G$. Let $\phi$ be an Orlicz function which satisfies the condition $\Delta^{\prime}$ globally with a constant $c>0$. Let us assume that for a Borel measurable function $h:[0, \infty) \rightarrow[0, \infty)$

$$
\text { there exists } \lambda>0 \text { such that } h(x) \leq \phi^{-1}(\lambda x) \text { for } x \in[0, \infty) \text {. }
$$

Then $T_{\tau}^{(\phi, h)}$ is a bounded linear operator on the Orlicz space $L^{\phi}(m)$ and

$$
\left\|T_{\tau}^{(\phi, h)}\right\|_{\phi} \leq \max \{1, c \lambda\} .
$$

Proof. It is clear that formula (2) gives a linear operator. Let $f \in L^{\phi}(m)$ and $\|f\|_{\phi}=1$. Let $d=\max \{1, c \lambda\}$. We obtain

$$
\begin{aligned}
& \int_{[0,1]} \phi \circ\left|T_{\tau}^{(\phi, h)}\left(\frac{f}{d}\right)\right| \mathrm{d} m=\int_{[0,1]} \phi \circ \frac{\left(|f| \circ \tau^{-1}\right)\left(h \circ \omega_{\tau}\right)}{d} \mathrm{~d} m \leq \text { (by the } \Delta^{\prime} \text { condition) } \\
& \left.c \int_{[0,1]}\left(\phi \circ \frac{|f| \circ \tau^{-1}}{d}\right)\left(\phi \circ h \circ \omega_{\tau}\right) \mathrm{d} m \leq \text { (the assumption on } h\right) \\
& \left.c \int_{[0,1]}\left(\phi \circ \frac{|f| \circ \tau^{-1}}{d}\right) \lambda \omega_{\tau} \mathrm{d} m \leq \text { (since } \phi \text { is an Orlicz function and } 1 \leq d\right) \\
& \frac{c \lambda}{d} \int_{[0,1]}\left(\phi \circ|f| \circ \tau^{-1}\right) \omega_{\tau} \mathrm{d} m=\text { (change of variables) } \frac{c \lambda}{d} \int_{[0,1]}(\phi \circ|f|) \mathrm{d} m \leq \\
& \text { (since } \left.\left.\|f\|_{\phi}=1\right) \frac{c \lambda}{d} \leq \text { (the definition of } d\right) 1 .
\end{aligned}
$$

Therefore, $\left\|T_{\tau}^{(\phi, h)}\left(\frac{f}{d}\right)\right\|_{\phi} \leq 1,\left\|T_{\tau}^{(\phi, h)}(f)\right\|_{\phi} \leq d$ and $\left\|T_{\tau}^{(\phi, h)}\right\|_{\phi} \leq d$.

Proposition 2.3. Let $\phi$ be an Orlicz function. Let us assume that for a Borel measurable function $h:[0, \infty) \rightarrow[0, \infty)$

$$
\text { there exists } \eta>0 \text { such that } x \leq \phi(\eta h(x)) \text { for } x \in[0, \infty) \text {. }
$$

Then $\left\|T_{\tau}^{(\phi, h)}\right\|_{\phi} \geq \frac{\phi^{-1}(1)}{\eta}$ for $\tau \in G$. 
Proof. We obtain $\int \phi \circ T_{\tau}^{(\phi, h)}\left(\eta \chi_{[0,1]}\right) \mathrm{d} m=\int \phi \circ\left(\eta h \circ \omega_{\tau}\right) \mathrm{d} m \geq \int \omega_{\tau} \mathrm{d} m=1$. Therefore, $\left\|T_{\tau}^{(\phi, h)}\left(\eta \chi_{[0,1]}\right)\right\|_{\phi} \geq 1$ and $\left\|T_{\tau}^{(\phi, h)}\left(\phi^{-1}(1) \chi_{[0,1]}\right)\right\|_{\phi} \geq \frac{\phi^{-1}(1)}{\eta}$, which implies our assertion since $\left\|\phi^{-1}(1) \chi_{[0,1]}\right\|_{\phi}=1$.

COROLlary 2.4. Let $\phi$ be an Orlicz function which satisfies the condition $\Delta^{\prime}$ globally with a constant $c$, a function $h:[0, \infty) \rightarrow[0, \infty)$ be Borel measurable and both conditions (3), (4) hold. Then

$$
\frac{\phi^{-1}(1)}{\eta} \leq\left\|T_{\tau}^{(\phi, h)}\right\|_{\phi} \leq \max \{1, c \lambda\}
$$

for $\tau \in G$.

3. Two approaches to coarse topologies. We recall the definition of the strong operator topology.

Definition 3.1. If $E, F$ are normed linear spaces then the strong operator topology on the set $\mathcal{L}(E, F)$ of bounded linear operators is given by the basis consisting of the elements $V\left(P, \varepsilon ; x_{1}, \ldots, x_{n}\right)=\left\{Q \in \mathcal{L}(E, F):(\forall i \in\{1, \ldots, n\})\left\|P\left(x_{i}\right)-Q\left(x_{i}\right)\right\|<\varepsilon\right\}$, where $P \in \mathcal{L}(E, F)$ is a bounded linear operator, vectors $x_{1}, \ldots, x_{n} \in E$ and $\varepsilon>0$.

Remark 3.2. Whenever space $E$ is separable and $A \subseteq \mathcal{L}(E, F)$ is a bounded set (that is, $(\exists M>0)(\forall P \in A)\|P\| \leq M)$, the strong operator topology on $A$ is metrizable by

$$
d(P, Q)=\sum_{n \in \mathbb{N}} \frac{\left\|P\left(f_{n}\right)-Q\left(f_{n}\right)\right\|}{2^{n}\left\|f_{n}\right\|} \text { for } P, Q \in A,
$$

where $\left\{f_{n} \in E \backslash\{0\}: n \in \mathbb{N}\right\}$ is a fixed countable dense subset of $E$.

If $\phi, h$ are as in Proposition 2.2 then the Orlicz space $E=L^{\phi}(m)$ is separable, the set $G_{\phi, h}=\left\{T_{\tau}^{(\phi, h)}: \tau \in G\right\}$ is bounded in the space $\mathcal{L}\left(L^{\phi}(m)\right)$ and the strong operator topology on $G_{\phi, h}$ is metrizable by the above metric.

Let us notice that the set $G_{\phi, h}$ is the image of the map

$$
T^{(\phi, h)}: G \ni \tau \mapsto T^{(\phi, h)}(\tau):=T_{\tau}^{(\phi, h)} \in \mathcal{L}\left(L^{\phi}(m)\right) .
$$

We are ready to prove the following lemma. If $S \subseteq[0,1]$ then $\chi_{S}$ denotes the characteristic function of $S$.

Lemma 3.3. Let $\phi$ be an Orlicz function which satisfies the condition $\Delta^{\prime}$ globally. Let a Borel measurable function $h:[0, \infty) \rightarrow[0, \infty)$ be nonzero on $(0, \infty)$ and let there exist $\lambda>0$ such that $h(x) \leq \phi^{-1}(\lambda x)$ for $x \in[0, \infty)$. Then the map $T^{(\phi, h)}: G \rightarrow \mathcal{L}\left(L^{\phi}(m)\right)$ is an injection.

Proof. Let us consider any two transformations $\tau \neq \sigma \in G$. There exists a Lebesgue measurable subset $A \subseteq[0,1]$ such that $m(\tau(A) \triangle \sigma(A)) \neq 0$, where $\triangle$ denotes the symmetric difference of sets. We obtain $T_{\tau}^{(\phi, h)}\left(\chi_{A}\right)=\chi_{\tau(A)}\left(h \circ \omega_{\tau}\right) \neq \chi_{\sigma(A)}\left(h \circ \omega_{\sigma}\right)=T_{\sigma}^{(\phi, h)}\left(\chi_{A}\right)$ since $h\left(\omega_{\tau}(x)\right), h\left(\omega_{\sigma}(x)\right) \neq 0$ almost everywhere.

We define coarse topologies on $G$.

Definition 3.4. Let $\phi$ be an Orlicz function which satisfies the condition $\Delta^{\prime}$ globally with a constant $c>0$. Let a function $h:[0, \infty) \rightarrow[0, \infty)$ be Borel measurable and let 
there exist $\lambda>0$ such that $h(x) \leq \phi^{-1}(\lambda x)$ for $x \in[0, \infty)$. We will denote by $\Theta_{\phi, h}$ the topology on the group $G$ which is induced from the strong operator topology on $G_{\phi, h}$ by the map $T^{(\phi, h)}$.

COROLlary 3.5. Let $\phi$ be an Orlicz function which satisfies the condition $\Delta^{\prime}$ globally. Let a Borel measurable function $h:[0, \infty) \rightarrow[0, \infty)$ be nonzero on $(0, \infty)$ and let there exist $\lambda>0$ such that $h(x) \leq \phi^{-1}(\lambda x)$ for $x \in[0, \infty)$. Then the topology $\Theta_{\phi, h}$ on the group $G$ is metrizable.

Proof. The assertion follows from Lemma 3.3 and Remark 3.2 .

We need the following result from general measure theory.

Lemma 3.6. Let $(\Omega, \Sigma)$ be a measurable space and let $\mu, \nu$ be measures on $\Sigma$, where $\mu$ is finite. If $\mu$ is absolutely continuous with respect to $\nu$ (that is $\nu(S)=0$ implies $\mu(S)=0$ ) and $\lim _{n \rightarrow \infty} \nu\left(S_{n}\right)=0$ for a sequence $\left\{S_{n}\right\} \subseteq \Sigma$ then $\lim _{n \rightarrow \infty} \mu\left(S_{n}\right)=0$.

Proof. The assertion follows from [H], Sec. 30, Theorem B.

Corollary 3.7. If $\tau \in G$ and $\left\{S_{n}: n \in \mathbb{N}\right\}$ is a sequence of Lebesgue measurable subsets of $[0,1]$ then

$$
\lim _{n \rightarrow \infty} m\left(\tau\left(S_{n}\right)\right)=0 \text { iff } \lim _{n \rightarrow \infty} m\left(S_{n}\right)=0 \text { iff } \lim _{n \rightarrow \infty} m\left(\tau^{-1}\left(S_{n}\right)\right)=0 .
$$

Moreover, if $\phi$ is an Orlicz function, the above equivalences can be continued:

$$
\lim _{n \rightarrow \infty} m\left(S_{n}\right)=0 \text { iff } \lim _{n \rightarrow \infty}\left\|\chi_{S_{n}}\right\|_{\phi}=0 \text { iff } \lim _{n \rightarrow \infty}\left\|\chi_{\tau\left(S_{n}\right)}\right\|_{\phi}=0 \text { iff } \lim _{n \rightarrow \infty}\left\|\chi_{\tau^{-1}\left(S_{n}\right)}\right\|_{\phi}=0 \text {. }
$$

Proof. The first two equivalences are consequences of Lemma 3.6. The second sequence of equivalences follows from the equality $\left\|\chi_{S}\right\|_{\phi}=\frac{1}{\phi^{-1}(1 / m(S))}$, which holds for any Lebesgue measurable subset $S \subseteq[0,1]$ of nonzero measure $m(S) \neq 0$.

We present another approach to the topology we are interested in, based on the ideas from [G1] and then continued in [B2].

Definition 3.8. Let $\Xi_{\phi, h}$ denote the topology on the group $G$ which is generated by the base consisting of the following sets:

$$
\begin{aligned}
U_{h}\left(\tau, \varepsilon,\left(I_{i}\right)_{i=1}^{n}\right)=\left\{\sigma \in G:(\forall k \in\{1, \ldots, n\})\left[m \left(\tau\left(I_{k}\right)\right.\right.\right. & \left.\triangle \sigma\left(I_{k}\right)\right)<\varepsilon \\
& \left.\left.\wedge\left\|h \circ \omega_{\tau}-h \circ \omega_{\sigma}\right\|_{\phi}<\varepsilon\right]\right\},
\end{aligned}
$$

where $\tau \in G, \varepsilon>0$ is rational and $\left(I_{k}\right)_{k=1}^{n}$ is a finite sequence of subintervals of $[0,1]$ such that the ends of each $I_{k}$ are rational.

We can now formulate the following theorem.

TheOREm 3.9. Let $\phi$ be an Orlicz function which satisfies the condition $\Delta^{\prime}$ globally. Let a Borel measurable function $h:[0, \infty) \rightarrow[0, \infty)$ be nonzero on $(0, \infty)$ and there exist $\lambda>0$ such that $h(x) \leq \phi^{-1}(\lambda x)$ for $x \in[0, \infty)$. Then $\Xi_{\phi, h}=\Theta_{\phi, h}$.

Proof. Since the topology $\Theta_{\phi, h}$ is metrizable (by Corollary 3.5 and the topology $\Xi_{\phi, h}$ is defined by a countable local basis at every invertible transformation, it is enough to show that $\lim _{n \rightarrow \infty} \tau_{n}=\tau$ in $\Theta_{\phi, h}$ iff $\lim _{n \rightarrow \infty} \tau_{n}=\tau$ in $\Xi_{\phi, h}$ for any transformations $\tau, \tau_{1}, \ldots, \tau_{n}, \ldots \in G$. 
(i) First, let us assume that $\lim _{n \rightarrow \infty} \tau_{n}=\tau$ in $\Theta_{\phi, h}$. Since $U\left(\sigma, \varepsilon, I_{1}, \ldots, I_{m}\right)=$ $\bigcap_{k=1}^{m} U\left(\sigma, \varepsilon, I_{k}\right)$, to prove that $\lim _{n \rightarrow \infty} \tau_{n}=\tau$ in $\Xi_{\phi, h}$ it is enough to show that the following two conditions hold:

(a) $\lim _{n \rightarrow \infty}\left\|h \circ \omega_{\tau_{n}}-h \circ \omega_{\tau}\right\|_{\phi}=0$ and

(b) $\lim _{n \rightarrow \infty} m\left(\tau_{n}(I) \triangle \tau(I)\right)=0$ for every subinterval $I \subseteq[0,1]$.

For (a), we observe that $\left\|h \circ \omega_{\tau_{n}}-h \circ \omega_{\tau}\right\|_{\phi}=\left\|T_{\tau_{n}}\left(\chi_{[0,1]}\right)-T_{\tau}\left(\chi_{[0,1]}\right)\right\|_{\phi} \rightarrow 0$.

For (b), let $S_{n}=\tau_{n}(I) \triangle \tau(I)$. First we claim that $\lim _{n \rightarrow \infty} \int_{S_{n}} h \circ \omega_{\tau} \mathrm{d} m=0$. We have

$$
\begin{aligned}
& \int_{S_{n}} h \circ \omega_{\tau} \mathrm{d} m \\
& =\int_{[0,1]}\left|\chi_{\tau_{n}(I)}-\chi_{\tau(I)}\right| h \circ \omega_{\tau} \mathrm{d} m=\int_{[0,1]}\left|\chi_{\tau_{n}(I)} h \circ \omega_{\tau}-\chi_{\tau(I)} h \circ \omega_{\tau}\right| \mathrm{d} m \\
& \leq \int_{[0,1]}\left|\chi_{\tau_{n}(I)} h \circ \omega_{\tau}-\chi_{\tau_{n}(I)} h \circ \omega_{\tau_{n}}\right| \mathrm{d} m+\int_{[0,1]}\left|\chi_{\tau_{n}(I)} h \circ \omega_{\tau_{n}}-\chi_{\tau(I)} h \circ \omega_{\tau}\right| \mathrm{d} m \\
& =\int_{[0,1]} \chi_{\tau_{n}(I)}\left|T_{\tau_{n}}^{(\phi, h)}\left(\chi_{[0,1]}\right)-T_{\tau}^{(\phi, h)}\left(\chi_{[0,1]}\right)\right| \mathrm{d} m+\int_{[0,1]}\left|T_{\tau_{n}}^{(\phi, h)}\left(\chi_{I}\right)-T_{\tau}^{(\phi, h)}\left(\chi_{I}\right)\right| \mathrm{d} m \\
& \leq \int_{[0,1]}\left|T_{\tau_{n}}^{(\phi, h)}\left(\chi_{[0,1]}\right)-T_{\tau}^{(\phi, h)}\left(\chi_{[0,1]}\right)\right| \mathrm{d} m+\int_{[0,1]}\left|T_{\tau_{n}}^{(\phi, h)}\left(\chi_{I}\right)-T_{\tau}^{(\phi, h)}\left(\chi_{I}\right)\right| \mathrm{d} m \\
& \leq\left(\mathrm{by} \mathrm{Jensen's} \mathrm{inequality)} \phi^{-1}\left(\int_{[0,1]} \phi \circ\left(T_{\tau_{n}}^{(\phi, h)}\left(\chi_{[0,1]}\right)-T_{\tau}^{(\phi, h)}\left(\chi_{[0,1]}\right)\right) \mathrm{d} m\right)\right. \\
& \quad+\phi^{-1}\left(\int_{[0,1]} \phi \circ\left(T_{\tau_{n}}^{(\phi, h)}\left(\chi_{I}\right)-T_{\tau}^{(\phi, h)}\left(\chi_{I}\right)\right) \mathrm{d} m\right) .
\end{aligned}
$$

The last two terms tend to 0 because norm convergence implies modular convergence and $\phi^{-1}(x) \rightarrow 0$ when $x \rightarrow 0^{+}$.

To finish this part of the proof, we show that $m\left(S_{n}\right)$ tends to 0 . Let us define the measure $m_{\tau, h}$ by the formula $m_{\tau, h}(S):=\int_{S} h \circ \omega_{\tau}=0$ for Lebesgue measurable subsets $S \subseteq[0,1]$. If $m_{\tau, h}(S)=0$ then $h\left(\omega_{\tau}(x)\right)=0$ for $m$-almost all $x \in S, \omega_{\tau}(x)=0$ for $m$-almost all $x \in S, m(\tau(S))=\int_{S} \omega_{\tau}=0$ and, by nonsingularity of invertible transformations, $m(S)=0$. This means that $m$ is absolutely continuous with respect to $m_{\tau, h}$. Finally, the convergence $m_{\tau, h}\left(S_{n}\right) \rightarrow 0$ implies $m\left(S_{n}\right) \rightarrow 0$ by Lemma 3.6.

(ii) For the opposite direction, let $\lim _{n \rightarrow \infty} \tau_{n}=\tau$ in $\Xi_{\phi, h}$. Since $V\left(\tau, \varepsilon, f_{1}, \ldots, f_{m}\right)=$ $\bigcap_{k=1}^{m} V\left(\tau, \varepsilon, f_{k}\right)$, it is enough to show that $\lim _{n \rightarrow \infty}\left\|T_{\tau_{n}}^{(\phi, h)}(f)-T_{\tau}^{(\phi, h)}(f)\right\|_{\phi}=0$ for every $f \in \mathcal{L}\left(L^{\phi}(m)\right)$.

(a) Assume that $f=\chi_{I}, I=[\alpha, \beta]$, where $0 \leq \alpha<\beta \leq 1$. Let $S_{n}=\tau_{n}(I) \triangle \tau(I)$. We obtain

$$
\begin{aligned}
\left\|T_{\tau_{n}}^{(\phi, h)}\left(\chi_{I}\right)-T_{\tau}^{(\phi, h)}\left(\chi_{I}\right)\right\|_{\phi} & =\left\|\chi_{\tau_{n}(I)} h \circ \omega_{\tau_{n}}-\chi_{\tau(I)} h \circ \omega_{\tau}\right\|_{\phi} \\
& \leq\left\|\chi_{\tau_{n}(I)}\left(h \circ \omega_{\tau_{n}}-h \circ \omega_{\tau}\right)\right\|_{\phi}+\left\|h \circ \omega_{\tau}\left(\chi_{\tau_{n}(I)}-\chi_{\tau(I)}\right)\right\|_{\phi} \\
& \leq\left\|h \circ \omega_{\tau_{n}}-h \circ \omega_{\tau}\right\|_{\phi}+\left\|h \circ \omega_{\tau} \chi_{S_{n}}\right\|_{\phi} .
\end{aligned}
$$

The first term tends to 0 because $\tau_{n}$ tends to $\tau$ in $\Xi_{\phi, h}$. 
For the second term,

$$
\left\|h \circ \omega_{\tau} \chi_{S_{n}}\right\|_{\phi}=\left\|T_{\tau}^{(\phi, h)}\left(\chi_{\tau\left(S_{n}\right)}\right)\right\|_{\phi} \leq\left\|T_{\tau}^{(\phi, h)}\right\|_{\phi}\left\|\chi_{\tau\left(S_{n}\right)}\right\|_{\phi} \leq d\left\|\chi_{\tau\left(S_{n}\right)}\right\|_{\phi},
$$

where $\left\|T_{\tau}^{(\phi, h)}\right\|_{\phi} \leq d$ as in Proposition 2.2

Since $m\left(S_{n}\right)$ tends to $0,\left\|\chi_{\tau\left(S_{n}\right)}\right\|_{\phi}$ tends to 0 by Corollary 3.7

(b) We assume that $f \in L^{\phi}(m)$ is an arbitrary function. Let $g=\sum_{k=1}^{m} \alpha_{k} \chi_{I_{k}}$ be any simple function, where $I_{k}$ denotes a subinterval of $[0,1]$. Then

$$
\begin{aligned}
\left\|T_{\tau_{n}}^{(\phi, h)}(f)-T_{\tau}^{(\phi, h)}(f)\right\|_{\phi} \leq\left\|T_{\tau_{n}}^{(\phi, h)}(f-g)\right\|_{\phi}+\left\|T_{\tau_{n}}^{(\phi, h)}(g)-T_{\tau}^{(\phi, h)}(g)\right\|_{\phi} \\
+\left\|T_{\tau}^{(\phi, h)}(f-g)\right\|_{\phi} \leq 2 d\|f-g\|_{\phi}+\sum_{k=1}^{m}\left|\alpha_{k}\right|\left\|\left(T_{\tau_{n}}^{(\phi, h)}-T_{\tau}^{(\phi, h)}\right) \chi_{I_{k}}\right\|_{\phi} .
\end{aligned}
$$

Since $\|f-g\|_{\phi}$ can be smaller than any number $\varepsilon>0$ and, by (a), the sum in the last term tends to 0 as $n$ tends to infinity, the proof has been completed.

4. Equality of topologies. We formulate our main result.

MaIn TheOrem 4.1. Coarse topologies $\Theta_{\phi, h}$ on $G$ coincide for all Orlicz functions $\phi$ which satisfy the condition $\Delta^{\prime}$ globally and for all Borel measurable functions $h:[0, \infty) \rightarrow[0, \infty)$ such that $h(0)=0$ and the following two conditions hold:

$$
\begin{aligned}
& (\exists \lambda>0)(\forall x, y \in[0, \infty))|\phi(h(x))-\phi(h(y))| \leq \lambda|x-y|, \\
& (\exists \eta>0)(\forall x, y \in[0, \infty))\left|\phi^{-1}(x)-\phi^{-1}(y)\right| \leq \eta|h(x)-h(y)| .
\end{aligned}
$$

Proof. The equality $h(0)=0$ and assumption (5) (when $y=0$ ) imply $h(x) \leq \phi^{-1}(\lambda x)$ for $x \in[0, \infty)$. Since $\phi^{-1}$ is injective, property (6) shows that $h$ is injective as well and $h(x) \neq 0$ for $x>0$. Therefore, the results from previous sections can be applied.

Let $\phi, h$ be any pair of functions which satisfy the assumptions of this theorem. We show that $\Theta_{\phi, h}=\Theta_{1}$ or equivalently, $\Xi_{\phi, h}=\Xi_{1}$, where $\Theta_{1}:=\Theta_{\text {id,id }}, \Xi_{1}:=\Xi_{\text {id,id }}$ and $\operatorname{id}(x)=x$.

(i) We prove that $\Xi_{\phi, h} \subseteq \Xi_{1}$. By metrizability of these topologies, it is enough to show that if $\tau_{n}$ tends to $\tau$ in $\Xi_{1}$ then it tends to $\tau$ in $\Xi_{\phi, h}$.

The sequence $\left(\tau_{n}\right)$ tends to $\tau$ in $\Xi_{1}$ iff

(a) $\lim _{n \rightarrow \infty}\left\|\omega_{\tau_{n}}-\omega_{\tau}\right\|_{1}=0$ and

(b) $\lim _{n \rightarrow \infty} m\left(\tau_{n}(I) \triangle \tau(I)\right)=0$ for every subinterval $I \subseteq[0,1]$ of rational ends.

The sequence $\left(\tau_{n}\right)$ tends to $\tau$ in $\Xi_{\phi, h}$ iff

$\left(\mathrm{a}^{\prime}\right) \lim _{n \rightarrow \infty}\left\|h \circ \omega_{\tau_{n}}-h \circ \omega_{\tau}\right\|_{\phi}=0$

and the same condition (b) holds.

We show that (a) implies (a'). Let $\alpha_{n}=\left\|h \circ \omega_{\tau_{n}}-h \circ \omega_{\tau}\right\|_{\phi}$. If $\alpha_{n} \neq 0$ then

$$
\begin{aligned}
1 & =\int_{[0,1]} \phi \circ\left|\frac{1}{\alpha_{n}}\left(h \circ \omega_{\tau_{n}}-h \circ \omega_{\tau}\right)\right| \mathrm{d} m \leq\left(\text { by the } \Delta^{\prime} \text { condition }\right) \\
& \quad \int_{[0,1]} \phi\left(\frac{1}{\alpha_{n}}\right) \phi \circ\left|h \circ \omega_{\tau_{n}}-h \circ \omega_{\tau}\right| \mathrm{d} m \leq c \phi\left(\frac{1}{\alpha_{n}}\right) \int_{[0,1]}\left|\phi \circ h \circ \omega_{\tau_{n}}-\phi \circ h \circ \omega_{\tau}\right| \mathrm{d} m,
\end{aligned}
$$

where the last inequality holds since $\phi(|x-y|) \leq|\phi(x)-\phi(y)|$ for $x, y \geq 0$. 
We apply (5) and continue the above inequalities:

$$
1 \leq c \lambda \phi\left(\frac{1}{\alpha_{n}}\right) \int_{[0,1]}\left|\omega_{\tau_{n}}-\omega_{\tau}\right| \mathrm{d} m=c \lambda \phi\left(\frac{1}{\alpha_{n}}\right)\left\|\omega_{\tau_{n}}-\omega_{\tau}\right\|_{1} .
$$

Since $\left\|\omega_{\tau_{n}}-\omega_{\tau}\right\|_{1}$ tends to 0 and both $c, \lambda$ are constants, $\alpha_{n}$ tends to 0 as well.

(ii) We show that $\Xi_{1} \subseteq \Xi_{\phi, h}$. By Theorem 8 in [CK], $\Xi_{1}=\Xi_{p}$ for $1 \leq p<\infty$, where $\Xi_{p}=\Xi_{g, g^{-1}}$ for $g(x)=x^{p}$. Therefore, it is enough to show that $\Xi_{p} \subseteq \Xi_{\phi, h}$ for some $1<p<\infty$.

By Lemma 3.7 of $\mathrm{H}$. Hudzik in B2], since $\phi$ satisfies the condition $\Delta_{2}$ globally (a consequence of the $\Delta^{\prime}$ condition), there exists $1<p<\infty$ such that $\phi^{1 / p}(x+y) \leq$ $\phi^{1 / p}(x)+\phi^{1 / p}(y)$ for $x, y \geq 0$.

Let us notice that for such $p$ the following inequalities hold: $\left|\phi^{1 / p}(a)-\phi^{1 / p}(b)\right| \leq$ $\phi^{1 / p}(|a-b|)$ and $\left|\phi^{1 / p}(a)-\phi^{1 / p}(b)\right|^{p} \leq \phi(|a-b|)$ for $a, b \in[0, \infty)-$ it is enough to substitute $a=x+y$ and $b=y$ when $a \geq b$. We show that if $\left\|h \circ \omega_{\tau_{n}}-h \circ \omega_{\tau}\right\|_{\phi}$ tends to 0 then $\left\|\omega_{\tau_{n}}^{1 / p}-\omega_{\tau}^{1 / p}\right\|_{p}$ tends to 0 , which will complete our proof.

We obtain

$$
\begin{aligned}
& \left\|\omega_{\tau_{n}}^{1 / p}-\omega_{\tau}^{1 / p}\right\|_{p}^{p}=\int_{[0,1]}\left|\omega_{\tau_{n}}^{1 / p}-\omega_{\tau}^{1 / p}\right|^{p} \mathrm{~d} m \\
= & \int_{[0,1]}\left|\left(\phi \circ \phi^{-1} \circ \omega_{\tau_{n}}\right)^{1 / p}-\left(\phi \circ \phi^{-1} \circ \omega_{\tau}\right)^{1 / p}\right|^{p} \mathrm{~d} m \leq \int_{[0,1]} \phi \circ\left|\phi^{-1} \circ \omega_{\tau_{n}}-\phi^{-1} \circ \omega_{\tau}\right| \mathrm{d} m \\
& \leq\left(\text { by }[6) \int_{[0,1]} \phi \circ\left(\eta\left|h \circ \omega_{\tau_{n}}-h \circ \omega_{\tau}\right|\right) \mathrm{d} m .\right.
\end{aligned}
$$

Since $\left\|h \circ \omega_{\tau_{n}}-h \circ \omega_{\tau}\right\|_{\phi}$ tends to $0,\left\|\eta\left(h \circ \omega_{\tau_{n}}-h \circ \omega_{\tau}\right)\right\|_{\phi}$ tends to 0 as well. Finally, norm convergence implies modular convergence.

REMARK 4.2. The functions $h:[0, \infty) \rightarrow[0, \infty)$ above are continuous (since $\phi \circ h$ is continuous by (5)) and injective (by (6)). Since also $h(0)=0$, they are strictly increasing. Moreover, $\lim _{x \rightarrow \infty} h(x)=\infty$ (by (6), when $y=0$ ). They need not to be concave.

EXAMPLE 4.3. Let $\phi(x)=x^{2}$ and

$$
h(x)= \begin{cases}\sqrt{x} & \text { for } x \in[0,1] \cup(9, \infty) \\ \frac{1}{2} x+\frac{1}{2} & \text { for } x \in(1,4] \\ \frac{1}{10} x+\frac{21}{10} & \text { for } x \in(4,9] .\end{cases}
$$

Then $\phi$ and $h$ together satisfy the assumptions of Theorem 4.1 but $h$ is not concave since $h_{-}^{\prime}(9)=\frac{1}{10}<h_{+}^{\prime}(9)=\frac{1}{6}$.

REMARK 4.4. In Theorem 4.1, assumption (5) gives (when $y=0) h(x) \leq \phi^{-1}(\lambda x)$ for $x \in[0, \infty)$ and by Proposition $2.2 .\left\|T_{\tau}^{(\phi, h)}\right\|_{\phi} \leq \max \{1, c \lambda\}$.

On the other hand, assumption (6) gives (when $y=0) x \leq \phi(\eta h(x))$ for $0 \leq x<\infty$ and $\left\|T_{\tau}^{(\phi, h)}\right\|_{\phi} \geq \frac{\phi^{-1}(1)}{\eta}$, by Proposition 2.3 .

Acknowledgments. The authors thank Professor Zbigniew Lipecki for helpful remarks. 


\section{References}

[B1] M. Burnecki, Equality of coarse topologies in inverse transformations, Acta Univ. Carolin. Math. Ph. 37 (1996), no. 2, 3-5.

[B2] M. Burnecki, Invertible transformations acting on Orlicz spaces, Arch. Math. (Basel) 70 (1998), 319-330.

[B3] M. Burnecki, An operator characterization of $L^{p}$-spaces in a class of Orlicz spaces, in: Function Spaces VIII, Banach Center Publ. 79, Polish Acad. Sci. Inst. Math., Warsaw, 2008, 53-55.

[CK] J. R. Choksi, S. Kakutani, Residuality of ergodic measurable transformations and of ergodic transformations which preserve an infinite measure, Indiana Univ. Math. J. 28 (1979), 453-469.

[G1] R. Grząślewicz, Density theorems for measurable transformations, Colloq. Math. 48 (1984), 245-250.

[G2] R. Grząślewicz, Generating the group of transformations of [0,1], Arch. Math. (Basel) 49 (1987), 335-336.

[H] P. R. Halmos, Measure Theory, Grad. Texts Math. 18, Springer, New York, 1974.

[KR] M. A. Krasnosel'skii, Ya. B. Rutickii, Convex Functions and Orlicz Spaces, Noordhoff, Groningen, 1961.

[P] V. S. Prasad, Generating dense subgroups of measure preserving transformations, Proc. Amer. Math. Soc. 83 (1981), 286-288.

[RR] M. M. Rao, Z. D. Ren, Theory of Orlicz Spaces, Monogr. Textbooks Pure Appl. Math. 146, Marcel Dekker, New York, 1991. 